\title{
Efecto de la Alimentación con Pulpa de Café (Coffea arabica) en los Índices Productivos de Cuyes (Cavia porcellus L) Raza Perú
}

\author{
Effect of Coffee (Coffea arabica) Pulp Meal as Feed on Productive Parameters \\ of Guinea Pigs (Cavia porcellus L) - Peru Breed
}

\author{
Ives Yoplac ${ }^{1,2}$, Juan Yalta ${ }^{1}$, Héctor V. Vásquez ${ }^{1}$, Jorge L. Maicelo ${ }^{1}$
}

\section{Resumen}

El objetivo del estudio fue determinar el efecto de la alimentación con harina de pulpa de café (Coffea arabica) en los índices productivos de cuyes (Cavia porcellus L), raza Perú, durante la etapa de recría y engorde. Se trabajó con 35 cuyes hembras desde los 28 hasta los 91 días de edad, alimentados con dietas a base de alfalfa y concentrado (1:1). Los cuyes fueron distribuidos al azar en cinco tratamientos con inclusiones en el concentrado de $0,5,15,25$ y $35 \%$ de harina de pulpa de café. La adición de la harina de pulpa de café al concentrado no influyó en la ganancia de peso, conversión alimenticia y calidad sensorial (apariencia, olor, color de la carcasa y sabor de la carne), pero tuvo una relación inversa con el consumo alimenticio. Los mayores rendimientos de carcasa se obtuvieron hasta la inclusión de $25 \%$ de harina de pulpa de café en el concentrado. Por lo tanto, la harina de pulpa de café, como insumo para formular concentrados para cuyes, tiene potencial para incrementar los índices productivos.

Palabras clave: ganancia de peso; conversión alimenticia; rendimiento de carcasa; evaluación sensorial; concentrado; valor nutritivo

\section{Abstract}

The objective of this study was to determine the effect of feeding guinea pigs (Cavia porcellus $\mathrm{L}$ ) - Peru breed with coffee (Coffea arabica) pulp meal on productive parameters during the rearing and fattening stages. A total of 35 female guinea pigs were monitored from 28 to 91 days of age, and fed with alfalfa and concentrate (1:1). The animals were

\footnotetext{
${ }^{1}$ Instituto de Investigación en Ganadería y Biotecnología (IGBI), Laboratorio de Nutrición y Bromatología de Alimentos, Facultad de Ingeniería Zootecnista, Agronegocios y Biotecnología (FIZAB), Universidad Nacional Toribio Rodríguez de Mendoza de Amazonas (UNTRM), Chachapoyas, Perú

${ }^{2}$ E-mail: iyoplac.fizab@untrm.edu.pe
}

Recibido: 16 de noviembre de 2016

Aceptado para publicación: 14 de abril de 2017 
randomly distributed in five treatments with inclusions of $0,5,15,25$ and $35 \%$ of coffee pulp meal in the concentrate. The addition of the coffee pulp meal to the concentrate did not affect body weight gain, feed conversion and sensory quality (appearance, odor, carcass color and meat taste) but had an inverse relationship with feed consumption. The highest carcass yields were obtained with the inclusion of up to $25 \%$ of coffee pulp meal in the concentrate. Therefore, coffee pulp meal, as an input to formulate guinea pig concentrates, has the potential to increase the productive performance.

Key words: body weight gain; feed conversion; carcass yield; sensory evaluation; concentrate; nutritional value

\section{INTRODUCCIÓN}

El cobayo (Cavia porcellus L), cuy o cuye es un mamífero roedor nativo de América del Sur (Perú, Colombia, Bolivia, Ecuador) (Chauca, 1997). La producción de cuy en el Perú es importante por su alto valor nutricional en términos de contenido proteico y bajo contenido de colesterol y grasas (Vivas, 2013). Estas cualidades podrían contribuir a la seguridad alimentaria en zonas rurales, así como extender el mercado a escala comercial (Gil, 2007; Bazay et al., 2014). El INEI (2012), a través del IV Censo Nacional Agropecuario (CENAGRO), reportó una población total de 12.7 millones de cuyes, los cuales se encuentran mayormente en la zona de la sierra.

En zonas rurales, la alimentación del cuy es mayormente con deshechos de cocina y pasto como suplemento, lo que conduce a la obtención de bajos índices productivos (Meza et al., 2014). A nivel semicomercial se utiliza forraje verde como la alfalfa, maíz chala y la avena forrajera (Gil, 2007). Adicionalmente, para mejorar sus índices productivos, se utilizan concentrados que se elaboran con materias primas (maíz amarillo, torta de soya, minerales y vitaminas), cuyos precios se han incrementado en estos últimos años, encareciendo los costos de producción (Morales et al., 2011; Bazay et al., 2014). Este contexto exige el desarrollo de nuevas alternativas de alimentación que permitan obtener buenos índices productivos, sin incrementar los costos de producción.
Entre los estudios que evaluaron el efecto de diversos alimentos en la eficiencia productiva del cuy destaca el trabajo de Castillo et al. (2012), quienes lograron mejorar la productividad con el uso de forrajes y suplementos con bloques nutricionales. Quintana et al. (2013), asimismo, reportaron que la combinación de alfalfa verde, harina de cebada y bloque mineral mejoran la ganancia de peso, conversión alimenticia y rendimiento de la carcasa. Similares resultados se obtuvieron al combinar granos de cebada y semillas de girasol (Lozada et al., 2013), morera (Morus alba) como forraje verde y en harina de forraje (Apráez et al., 2008; Meza et al., 2014), ensilado de pescado (Mattos et al., 2003), gramíneas tropicales como pasto saboya (Panicum maximun), hojas de maíz y caña de azúcar (Sánchez et al., 2009), y forrajes tropicales y banano maduro (Sánchez et al., 2012).

La pulpa de café es el principal subproducto obtenido del procesamiento del grano de café y representa el $29 \%$ del total del fruto. La pulpa seca contiene $79.7 \mathrm{~g} \cdot \mathrm{kg}^{-1}$ de proteína cruda (PC), $35.7 \mathrm{~g} \cdot \mathrm{kg}^{-1}$ de fibra cruda (FC), $13.4 \mathrm{~g} \cdot \mathrm{kg}^{-1}$ de grasa y $5.38 \mathrm{MJ} \cdot \mathrm{kg}^{-1}$ de energía metabolizable (Donkoh et al., 1988) Estas propiedades le confieren cualidades nutritivas para ser utilizado en la alimentación animal, sea como ensilaje, torta de pulpa y secado para la obtención de harina (Noriega et al., 2008). No obstante, presentan otras sustancias que afectan su valor nutritivo, tales como taninos, cafeína y polifenoles libres, los cuales se encuentran en bajas con- 
centraciones, y que se pueden disminuir con procesos de fermentación, ensilaje y secado (Ramírez, 1988). Es importante mencionar que la región Amazonas es la principal productora de café orgánico en el Perú, certificado por IMOCert y Fairtrade Internacional, y donde la pulpa de café no es aprovechada como alimento animal, sino reciclada en los campos de cultivo (COOPARM, 2016).

Estudios previos reportan la utilización de hasta $9 \%$ de pulpa de café deshidratada en la alimentación de gallinas ponedoras y de engorde (Funes et al., 1986; Donkoh et al., 1988; Rodríguez et al., 1994), en alevines del híbrido cachamay (Colossoma $x$ Piaractus) y de tilapias (Moreau et al., 2003; Bautista et al., 2005), en ovinos (Ferreira et al., 2003) y en conejos (Duban et al., 2012). Sin embargo, no se ha encontrado evidencia del uso de la harina de pulpa de café en la alimentación de cuyes. Por tanto, el presente trabajo tuvo como objetivo evaluar el efecto de la alimentación con pulpa de café (Coffea arabica) en los índices productivos de cuyes (Cavia porcellus L), raza Perú.

\section{Materiales y Métodos}

\section{Lugar de Estudio}

El estudio se realizó en el Centro Experimental de Animales Menores del Instituto de Investigación en Ganadería y Biotecnología (IGBI) de la Facultad de Ingeniería Zootecnista, Agronegocios y Biotecnología (FIZAB) de la Universidad Nacional Toribio Rodríguez de Mendoza de Amazonas (UNTRM), Chachapoyas, Perú. El centro experimental se encuentra a 2335 msnm, y la zona presenta un clima templado moderadamente lluvioso, temperatura ambiental promedio de $18{ }^{\circ} \mathrm{C}$ y humedad relativa de $74 \%$.

\section{Animales}

Se utilizaron 35 cuyes (Cavia porcellus L) del IGBI, de la raza Perú en etapa de recría, con 28 días de edad, sexo hembras, con un peso entre 300 y $400 \mathrm{~g}$ y una talla entre 10 y $15 \mathrm{~cm}$. Los cuyes fueron distribuidos aleatoriamente en cinco pozas tecnificadas por un periodo de nueve semanas. Cada poza contenía siete cuyes identificados con aretes.

Los animales estuvieron en un galpón de paredes de ladrillo y concreto armado, techo de fibrocemento (Eternit) y ventanas con mallas metálicas finas, provistas de lunas transparentes para asegurar la luminosidad y ventilación. El área del galpón fue de $20 \times 8$ $\mathrm{m}$. Las pozas medían $1.50 \mathrm{~m}$ de largo x 0.75 $\mathrm{m}$ de ancho x $0.5 \mathrm{~m}$ de profundidad. Cada poza contenía un comedero tipo tolva de policloruro de vinilo (PVC) con capacidad de $2 \mathrm{~kg}$ y un bebedero de cemento con capacidad de 0.51 . Antes del inicio del experimento, el galpón y las pozas fueron limpiados y desinfectados con detergente y óxido de calcio, actividades que se repitieron cada 15 días. Los comederos y bebederos fueron lavados y desinfectados previo a cada entrega de alimento y bebida.

Los insumos que formaron parte del alimento concentrado, como la harina integral de soya, torta de soya $47 \%$ PC, maíz molido, aceite de palma, fosfato dicálcico, lisina y premezcla comercial (Pecutrin) fueron obtenidos de la Empresa Cogorno (Chiclayo, Perú). La pulpa de café fue obtenida en Vista Hermosa, provincia de Luya, región Amazonas.

\section{Harina de Pulpa de Café}

Los frutos se recolectaron con un índice de madurez óptimo, con coloración rojo oscuro. Se separó la pulpa del grano con despulpadoras artesanales. El grano, como producto principal, siguió operaciones espe- 
Cuadro 1. Composición porcentual de los concentrados utilizados en la etapa de recría y engorde de cuyes con inclusión de harina de pulpa de café (\%)

\begin{tabular}{lccccc}
\hline \multirow{2}{*}{ Ingredientes (\%) } & \multicolumn{5}{c}{ Concentrados (tratamientos) } \\
\cline { 2 - 6 } & $0 \%$ & $5 \%$ & $15 \%$ & $25 \%$ & $35 \%$ \\
\hline Harina integral de soya & 7.48 & 8.58 & 3.40 & 5.59 & 7.79 \\
Torta de soya 47\% PC & 4.92 & 3.86 & 7.50 & 5.39 & 3.27 \\
Maíz nacional & 35.78 & 30.73 & 20.27 & 10.17 & 0.07 \\
Aceite de palma & 0 & 0 & 2.00 & 2.00 & 2.00 \\
Heno de alfalfa molida & 50.00 & 50.00 & 50.00 & 50.00 & 50.00 \\
Harina de pulpa de café & 0.00 & 5.00 & 15.00 & 25.00 & 35.00 \\
Sal común & 0.37 & 0.37 & 0.373 & 0.37 & 0.37 \\
Fosfato dicálcico & 0.65 & 0.66 & 0.66 & 0.68 & 0.69 \\
Lisina (HCl 99\%) & 0.20 & 0.20 & 0.20 & 0.20 & 0.20 \\
Metionina & 0.31 & 0.31 & 0.31 & 0.30 & 0.30 \\
Premezcla comercial & 0.30 & 0.30 & 0.30 & 0.30 & 0.30 \\
\hline Total & 100.0 & 100.0 & 100.0 & 100.0 & 100.0 \\
\hline
\end{tabular}

cíficas para la obtención de café pergamino, no usado en esta investigación. En el residuo agroindustrial, cáscara y pulpa, se retiraron los restos de pedicelos, hojas y otros desechos, para luego envasarlo en bolsas de polipropileno (PP), y trasladarlo a las instalaciones de los laboratorios del IGBI en cajas de poliestireno expandido (tecnopor).

El residuo agroindustrial fue secado al sol hasta una humedad de $12 \%$. Se trituró en un molino de disco (Cemotec 1090, Foss, Dinamarca) hasta obtener harina con tamaño de partícula promedio de $250 \mu \mathrm{m}$. Luego fue envasado en bolsas de PP y almacenado en un ambiente con humedad relativa inferior a $70 \%$.

\section{Raciones Alimenticias}

En la formulación del concentrado para la etapa de recría y engorde se tomó en cuenta los requerimientos nutricionales recomenda- dos por Montes (2012). Se establecieron cinco tratamientos con la inclusión de la harina de pulpa de café en varios niveles: T0: testigo $(0 \%)$, T1: $5 \%$, T2: $15 \%$, T3: $25 \%$ y T4: $35 \%$ (Cuadro 1). La composición nutricional de cada ración se muestra en el Cuadro 2.

\section{Alimentación y Saca}

Los cuyes en la etapa de recría y engorde recibieron una alimentación mixta consistente en forraje $(50 \%)$ y concentrado $(50 \%)$, en base seca. El forraje a base de alfalfa se cortó y ventiló por 12 horas, antes del suministro a los cuyes. El alimento y agua fue dado ad libitum. El alimento fue proporcionado dos veces al día (07:00 y 17:00 h).

El beneficio de los cuyes se realizó a la novena semana del estudio siguiendo la metodología descrita por Morales et al. (2011). La carcasa estuvo compuesta por la cabeza, patas y el cuerpo sin vísceras, a excepción 
Cuadro 2. Composición nutricional de los concentrados utilizados en la etapa de recría y engorde de cuyes con inclusión de harina de pulpa de café (\%)

\begin{tabular}{lrrrrr}
\hline \multirow{2}{*}{ Componentes } & \multicolumn{5}{c}{ Concentrados (tratamientos) } \\
\cline { 2 - 6 } & $\mathrm{T} 0=0 \%$ & $\mathrm{~T} 1=5 \%$ & $\mathrm{~T} 2=15 \%$ & $\mathrm{~T} 3=25 \%$ & $\mathrm{~T} 4=35 \%$ \\
\hline Proteína cruda (\%) & 18.00 & 18.00 & 18.00 & 18.00 & 18.00 \\
Grasa cruda (\%) & 4.32 & 4.42 & 3.17 & 3.36 & 3.56 \\
Fibra cruda (\%) & 11.86 & 12.41 & 13.34 & 14.44 & 15.54 \\
FDN (\%) & 28.62 & 29.42 & 30.71 & 32.32 & 33.93 \\
Calcio (\%) & 1.12 & 1.14 & 1.19 & 1.24 & 1.30 \\
Fósforo disponible (\%) & 0.35 & 0.35 & 0.35 & 0.35 & 0.35 \\
Sodio (\%) & 0.20 & 0.20 & 0.20 & 0.20 & 0.20 \\
Cloro (\%) & 0.52 & 0.52 & 0.52 & 0.52 & 0.52 \\
Arginina (\%) & 0.90 & 0.90 & 0.89 & 0.88 & 0.87 \\
Lisina (\%) & 0.98 & 0.98 & 0.97 & 0.96 & 0.95 \\
Metionina + cisteína (\%) & 0.85 & 0.85 & 0.85 & 0.85 & 0.85 \\
Treonina (\%) & 0.70 & 0.70 & 0.70 & 0.70 & 0.70 \\
Triptófano (\%) & 0.22 & 0.22 & 0.22 & 0.22 & 0.21 \\
Vit. C extra (mg/100g) & 0.00 & 0.00 & 0.00 & 0.00 & 0.00 \\
Energía digestible (kcal/kg) & 2800 & 2800 & 2800 & 2800 & 2800 \\
\hline
\end{tabular}

de los riñones e hígado. La canal y las vísceras fueron pesadas por separado.

\section{Índices Productivos y Calidad Sensorial}

- Control de peso. Se realizó al inicio de experimento (28 días de edad) y cada 7 días, utilizando una balanza electrónica de $5 \mathrm{~kg}$ con $2 \mathrm{~g}$ de precisión (Tanita, España). Los animales fueron pesados a las 08:00 $\mathrm{h}$, antes del suministro del alimento.

- Ganancia de peso, consumo de alimento e indice de conversión alimenticia. Se empleó la metodología propuesta por Quintana et al. (2013). Los resultados se reportaron a $\operatorname{los} 35,42,63,77 \mathrm{y}$ 91 días de edad.

- Rendimiento de carcasa. Los animales fueron beneficiados previo ayuno de 24 horas, siguiendo el método descrito por Morales et al. (2011). Se emplearon dos cuyes por tratamiento. Para el peso de la carcasa se utilizó una balanza electrónica de $2 \mathrm{~kg}$ con $2 \mathrm{~g}$ de precisión (Tanita, España).

- Evaluación sensorial. Los cuyes beneficiados se emplearon, además, para la evaluación de calidad sensorial. Se utilizó el método de análisis descriptivocualitativo descrito por Char et al. (2016), aplicado a un panel de 10 jueces semientrenados, usando una pauta no estructurada de 0 a 15 , donde se evaluó el aspecto visual (apariencia e intensidad de color) y aspecto gustativo (olor y sabores extraños). En el caso de la apariencia e intensidad de color, los mayores valores indican mejor aceptabilidad, mientras que los menores valores para 
Cuadro 3. Composición química de la harina de pulpa de café (promedio \pm d.e.) $(\mathrm{n}=3)$

\begin{tabular}{lc}
\hline Componentes & Porcentaje \\
\hline Humedad & $10.09 \pm 0.02$ \\
Extracto etéreo & $3.48 \pm 0.07$ \\
Proteína & $14.03 \pm 0.09$ \\
Fibra cruda & $19.29 \pm 0.05$ \\
Cenizas & $11.95 \pm 0.14$ \\
Almidón & $13.92 \pm 0.07$ \\
Azúcar & $10.00 \pm 0.22$ \\
Fibra detergente neutro & $38.78 \pm 0.14$ \\
(FDN) & \\
Fibra detergente ácida & $26.64 \pm 0.07$ \\
(FDA) & \\
\hline
\end{tabular}

olor y sabores extraños indican mejor aceptabilidad. Las escalas usadas fueron: apariencia ( 0 : muy mala y 15 : extremadamente buena), intensidad de color (0: blanco y 15: extremadamente rosado), olor (0: no rancio y 15: extremadamente rancio) y sabores extraños $(0: \sin$ sabores extraños y 15: extremadamente presente), considerando una puntuación de 7.5 como media aceptable. Las unidades se consideraron como adimensionales.

A cada juez se le proporcionó las carcasas enteras por tratamiento entre la primera y segunda hora del beneficio. Además, se les proporcionó guantes quirúrgicos descartables solicitándoles el menor manipuleo posible con la finalidad de cuidar la higiene y de no afectar la calidad sensorial. Para la evaluación de sabores extraños se les proporción $5 \mathrm{~g}$ de carne magra, cruda, de la pierna derecha de cada cuy.
Mortalidad. Se contabilizó y registró diariamente. De haberse reportado bajas, se habría hecho la necropsia para establecer las posibles causas de la muerte.

\section{Diseño Experimental}

Se utilizó un diseño completamente al azar (DCA) con 5 tratamientos y 7 repeticiones por tratamiento. La unidad experimental correspondió a un cuy. Los resultados obtenidos de los índices productivos y la evaluación sensorial se evaluaron con un análisis de varianza al 5\% de significancia. Cuando se encontraron diferencias significativas se aplicó la prueba de comparación múltiple de Dunnett $(\alpha<0.05)$. Los datos se analizaron mediante el programa MINITAB v. 16.1 .

\section{Resultados y Discusión}

\section{Composición Química de la Pulpa de Café}

La harina de pulpa de café destacó en su concentración de fibra (19.29\%) y proteína (14.03\%) (Cuadro 3).

\section{Índices Productivos de los Cuyes}

Control de peso vivo

No se encontraron diferencias significativas entre el testigo y los tratamientos (Figura 1a), donde el peso inicial a los 28 días de edad fue de $368 \mathrm{~g}$ y los pesos a los 91 días de edad variaron entre 901 y 1075 g. Estos resultados fueron similares a los reportados por Apráez et al. (2008) y Quintana et al. (2013), pudiendo deberse a que la harina de pulpa de café presentó una disponibilidad adecuada y equilibrada de nutrientes (Montes, 2012). Cuando la dieta nutricional es deficiente en nutrientes digeribles ocasiona un deterioro de las funciones vitales, que se refleja principalmente en el peso del animal (Chauca, 1997), 
lo cual no ocurrió en la presente investigación.

\section{Ganancia de peso}

Al igual que en los resultados del control de peso, las ganancias de peso de los cuyes en todas las edades de evaluación, no presentaron diferencias significativas entre el testigo y los tratamientos (Figura 1b). La ganancia de peso al finalizar el estudio (91 días de edad) varió entre 65 y 85 g/día. Estos valores guardan relación con los reportados por Clemente et al. (2003); sin embargo, Mattos et al. (2003) obtuvieron mayores ganancias de peso con ensilado de pescado en relación a la torta de soya. Esto demuestra que las dietas elaboradas exclusivamente con insumos vegetales presentan una menor calidad proteica, la cual influye en la ganancia de peso. En el presente estudio la fuente proteica fue de origen vegetal.

En otros estudios, Quintana et al. (2013) obtuvieron mejores resultados cuando se usó harina de granos de cebada frente al uso de alfalfa y concentrado integral; en tanto que Lozada et al. (2013) obtuvieron mejores resultados al usar granos de cebada y girasol, debido a su aporte en almidón y lípidos, respectivamente.

Las ganancias de peso diarias por animal variaron entre 8.4 y $11.3 \mathrm{~g} \cdot \mathrm{dí}^{-1}$, valores superiores a los 4.4 - $9.1 \mathrm{~g} \cdot$ día $^{-1}$ encontrados por Meza et al. (2014) utilizando gramíneas y forrajes arbustivos (morera, caraca y cucarda), sin la adición de concentrados, y al $6.8 \mathrm{~g} \cdot \mathrm{día}^{-1}$ reportado por Sánchez et al. (2012), usando forrajes y banano maduro. Por el contrario, Apráez et al. (2008), al evaluar forrajes y alimentos no convencionales, obtuvieron valores superiores que variaron entre 11.7 y $12.3 \mathrm{~g} \cdot$ día, debido probablemente a que usaron alimentación equilibrada en la composición de aminoácidos. Estos resultados demuestran la importancia del uso de concentrados como complemento al uso de forrajes, así como, al origen de los insumos para elaboración de los concentrados (ani- mal o vegetal) y la composición nutricional de las raciones alimenticias.

\section{Consumo de alimento}

El consumo de alimento del testigo y la dieta con $5 \%$ de pulpa de café fueron estadísticamente superiores a los demás tratamientos en todas las evaluaciones (Figura 1c). A los 35 días de edad, las dietas con $0 \mathrm{y}$ $5 \%$ de pulpa de café presentaron consumos de 206 y 204 g, respectivamente, mientras que la dieta con mayor contenido de pulpa de café (35\%) presentó el menor consumo (178 g) $(\mathrm{p}<0.05)$. En forma similar, a los 91 días de edad, las dietas con 0 y $5 \%$ de pulpa de café obtuvieron 485 y $484 \mathrm{~g}$, respectivamente, valores superiores a la dieta con $35 \%$ de pulpa de café $(468 \mathrm{~g})(\mathrm{p}<0.05)$.

Los resultados muestran una relación inversa entre el consumo de alimento y el porcentaje de harina de pulpa de café, probablemente debido a que la aceptabilidad y la palatabilidad se reducen con la mayor inclusión de este insumo por el aumento de taninos, cafeína y polifenoles libres (ácido clorogénico y ácido cafeico), que influyen en el olor y sabor del concentrado (Noriega et al., 2008, 2009). Resultados con tendencia similar reportaron Funes et al. (1986), quienes emplearon pulpa de café deshidratada ente 0 y $30 \%$ en pollos de engorde; asimismo, Duban et al. (2012) obtuvieron mejores consumos en conejos con cáscara de cacao en relación a la dieta con pulpa de café, debido principalmente a su mejor palatabilidad. En general, se encuentra que el consumo de alimento se reduce cuando se suministra pulpa de café en concentraciones elevadas, tanto en rumiantes como en monogástricos (Braham y Bressani, 1990; Noriega et al., 2008).

Los consumos diarios promedio por cuy fueron de 49.2, 48.9, 48.4, 48.1 y $46.7 \mathrm{~g} \cdot$ día $^{-1}$ para los tratamientos con $0,5,12,25$ y $35 \%$ de pulpa de café, respectivamente. Estos resultados coinciden con los hallados por Mattos et al. (2003), aunque superiores a los observados por Meza et al. (2014) utilizando fo- 
rrajes arbustivos (Saboya, king-grass, maralfalfa, morera, cucarda y caraca) y por Ramos et al. (2013) suplementando con harina de nabo y forraje. Por el contrario, los resultados del presente estudio fueron inferiores a los consumos de 68.88 y $72.46 \mathrm{~g} \cdot \mathrm{dia}^{-1}$ encontrados por Lozada et al. (2013) utilizando granos de cebada y semillas de girasol, los 53.52 y $59.17 \mathrm{~g} \cdot \mathrm{día}^{-1} \cdot$ obtenidos por Apráez et al. (2008) utilizando alfalfa, morera y leucanena, y los 67.8 y $69.6 \mathrm{~g} \cdot$ día $^{-1}$ reportados por Sánchez et al. (2012) con forrajes tropicales y banano maduro; posiblemente debido a la mejor palatabilidad de estos insumos, así como a sus mejores características sensoriales.

Por lo tanto, es posible que para mejorar el grado de aceptabilidad de la harina de pulpa de café, se deba incluir en la dieta en mezcla con alimentos de alta palatabilidad, así como con forrajes y concentrados proteicos, a fin de utilizar este elemento como reemplazo de insumos tradicionales (Noriega et al., 2008).

\section{Conversión alimenticia}

No se observaron diferencias estadísticas entre el testigo y los tratamientos, con excepción a la edad de 77 días, donde la dieta con $35 \%$ de pulpa de café presentó una conversión alimenticia de 8.08 , en contraste con los demás tratamientos con valores entre 6.00 y 6.53 (Figura 1d). Los índices de conversión alimenticia, en todas las edades evaluadas, muestran una tendencia decreciente cuando se incrementa la harina de pulpa de café en el concentrado; no obstante, estos índices están dentro de los rangos sugeridos por Chauca (1997) y coinciden con los obtenidos por Mattos et al. (2003); Meza et al. (2014) y Ramos et al. (2013).

Los resultados del presente estudio muestran una mayor eficiencia de conversión alimenticia a los reportados en otros estudios. Así, Lozada et al. (2013) obtuvieron índices entre 8.04 y 10.88 al utilizar granos de cebada y semillas de girasol; Sánchez et al. (2009) obtuvieron índices de 13.8 alimentando con gramíneas tropicales a cuyes en etapa de engorde, y Sánchez et al. (2012) obtuvieron entre 7.55 y 12.17 alimentando con forraje y banano maduro. Por el contrario, Quintana et al. (2013) obtuvieron una conversión alimenticia de 3.0 y 5.7 usando alfal$\mathrm{fa}$, granos de cebada y bloques minerales, en tanto que Apráez et al. (2008) obtuvieron índices entre 4.35 y 5.02 alimentando con forrajes arbustivos.

El índice de conversión alimenticia valora indirectamente el uso del alimento en base a su digestibilidad, absorción y calidad de nutrientes (Mattos et al., 2003). Con los resultados obtenidos en el presente estudio, incluidos la ganancia de peso, se podría afirmar que el incremento de la harina de pulpa de café en la dieta experimental afecta estos indicadores productivos. Por otro lado, este índice está en función de la composición proteica y aporte energético de la dieta, de allí que dietas iguales e inferiores a $25 \%$ de harina de pulpa de café cubrieron los requerimientos nutricionales sin afectar la conversión alimenticia.

Ganancia de peso, consumo de alimento y conversión alimenticia total

La ganancia de peso fue entre de 0.53 y $0.71 \mathrm{~kg}$ y la conversión alimenticia total varió entre 4.44 y 5.60 al final del estudio (91 días de edad), sin que se observen diferencias significativas entre tratamientos. Por el contrario, el consumo alimenticio total del testigo fue significativamente superior $(3.10 \mathrm{~kg})$ en relación a los tratamientos con pulpa de café (2.94-3.08 kg) $(\mathrm{p}<0.05$; Figura $2 \mathrm{a})$; y con valores similares a los reportados por Carbajal (2015).

Los resultados obtenidos fueron mejores a los hallados en otros estudios donde se evaluaron forrajes de maíz y bloques minerales (Castillo et al., 2012), alfalfa, cebada y bloque minerales (Quintana et al., 2013), y antibióticos promotores de crecimiento y aditivos de manano-oligosacáridos (Bazay et al., 

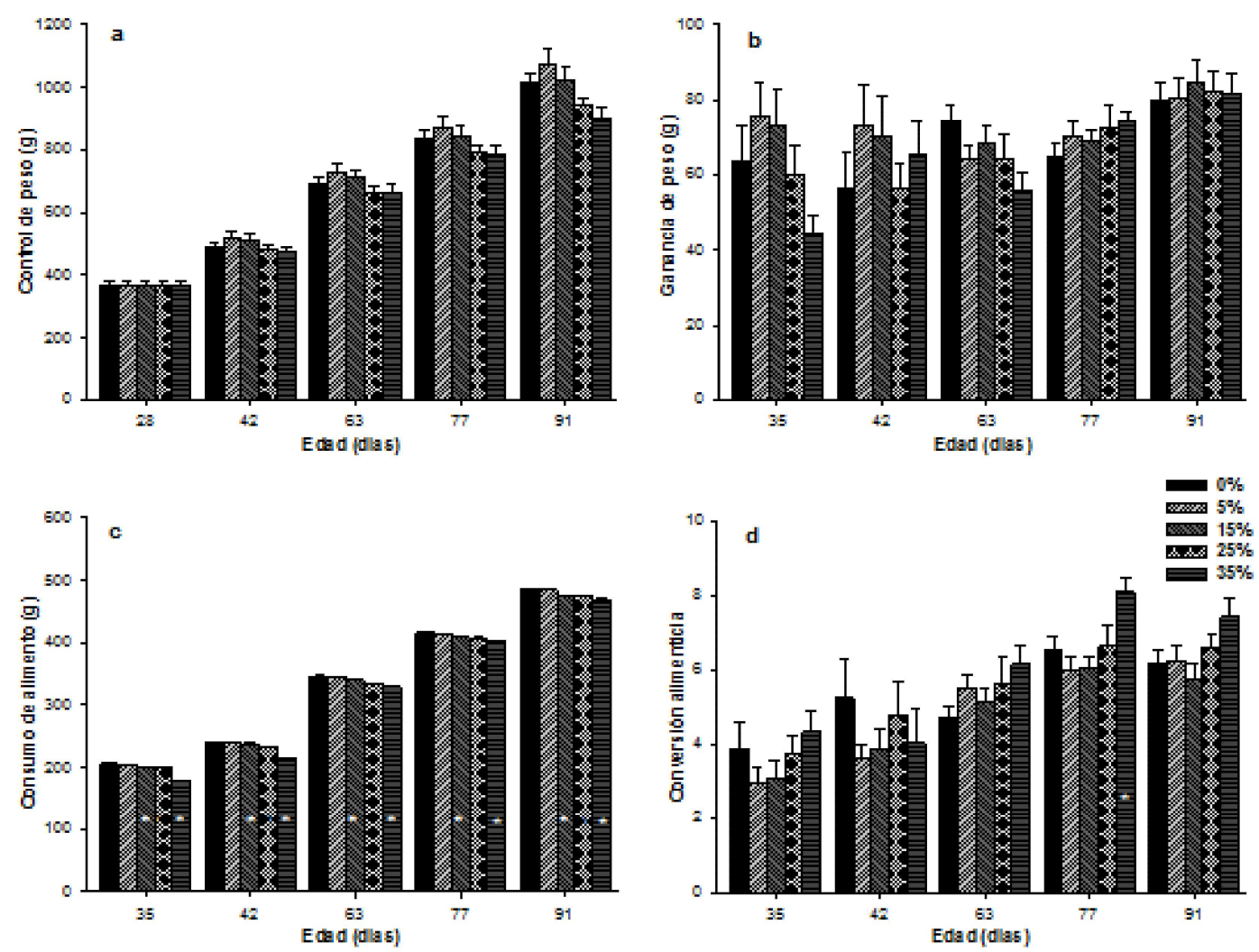

Figura 1. Control de peso (a); ganancia de peso (b); consumo de alimento (c) e índice de conversión alimenticia (d) de cuyes $(\mathrm{n}=7$ por tratamiento) alimentados con niveles crecientes de harina de pulpa de café desde los 28 días hasta los 91 días de edad. Las barras indican el error estándar. * indica diferencias significativas entre tratamientos y el testigo $(\mathrm{p}<0.05)$.

2014). Estas diferencias podrían estar vinculadas a las características de las dietas y la composición equilibrada del concentrado alimenticio, el estado fisiológico y genético de los animales, y las condiciones del manejo como temperatura ambiental, humedad relativa, hacinamiento y sanidad (Bazay et al., 2014). En el presente estudio, se empleó una dosificación adecuada de nutrientes en las raciones alimenticias que incluía el aporte proteico, energético y en fibra de la harina de pulpa de café. Además, se emplearon cuyes mejorados y se llevó a cabo en condiciones ambientales e higiénicas adecuadas, lo que podría haber influido en la obtención de respuestas productivas positivas.

\section{Rendimiento de carcasa}

Los rendimientos de carcasa del testigo y las dietas con 5,15 y $25 \%$ de harina de pulpa de café presentaron valores entre 70.8 y $71.5 \%$, mientras que la dieta con $35 \%$ de harina de pulpa de café presentó un rendimiento de $68.1 \%(p<0.05$; Figura $2 b)$. No obstante, todos los rendimientos fueron superiores al promedio sugerido para cuyes mejorados en pozas de $67.4 \%$ (Chauca, 1997); dado que las dietas utilizadas poseen adecuados niveles de proteína, energía, vita- 

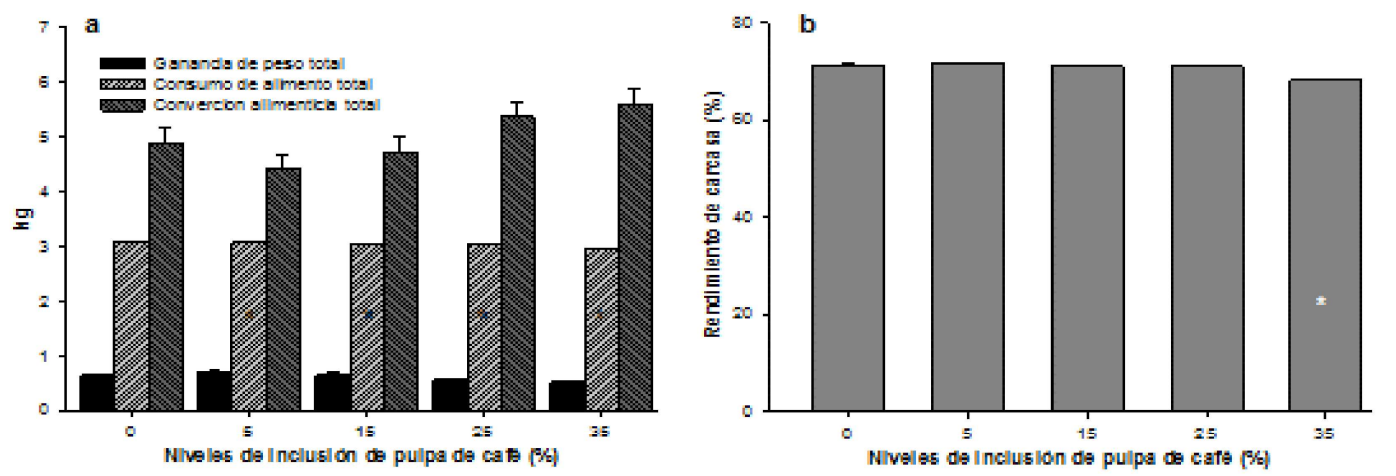

Figura 2. Ganancia de peso total, consumo de alimento total y conversión alimenticia total (a), y rendimiento de carcasa (b) de cuyes alimentados con niveles crecientes de harina de pulpa de café desde los 28 días hasta la edad de 91 días. (a) Las barras indican el error estándar ( $\mathrm{n}=7$ por tratamiento) y (b) las barras indican el error estándar ( $\mathrm{n}=2$ por tratamiento). ${ }^{*}$ indican diferencias significativas entre los tratamientos y el testigo $(\mathrm{p}<0.05)$

minas y minerales, que permiten cubrir los requerimientos de la especie (Montes, 2012).

Los rendimientos de carcasa en este estudio fueron similares a otros (Mattos et al., 2003; Morales et al., 2011, Sánchez et al., 2012; Ramos et al., 2013), aunque los obtenidos con las dietas de 0 a $25 \%$ de harina de pulpa de café superan al rendimiento de carcasa de 62.7 y $69.9 \%$ reportado por Meza et al. (2014) en cuyes alimentados con gramíneas y forrajes arbustivos tropicales, $\mathrm{y}$ del 64.9-67.4\% de Apráez et al. (2008) en cuyes alimentados con alfalfa, morera y leucaena.

\section{Evaluación sensorial}

La apariencia, olor y sabor de la carcasa del cuy no presentaron diferencias significativas entre el testigo y los tratamientos. La apariencia varió entre 9.29 y 10.76 puntos, el olor entre 0.31 y 0.58 puntos y el sabor entre 0.46 y 0.71 puntos.
Al evaluar el color de la carcasa, la dieta con $15 \%$ de harina de pulpa de café obtu vo 9.59 puntos, valor significativamente superior al testigo y demás tratamientos que variaron entre 7.63 y 8.95 puntos (Cuadro 4). Los valores de calidad sensorial encontrados en la presente investigación fueron aceptables, observando que la harina de pulpa de café no influyó significativamente en estos parámetros. Resultados similares fueron reportados por Mattos et al. (2003).

\section{Mortalidad}

No ocurrieron muertes en ninguno de los grupos experimentales.

\section{Conclusiones}

- La inclusión de harina de pulpa de café en la formulación del concentrado alimenticio de cuyes no influyó en la ga- 
Cuadro 4. Evaluación sensorial de la carcasa de cuyes alimentados con forraje y concentrado con niveles crecientes de harina de pulpa de café (promedio \pm e.e.) $(\mathrm{n}=10)$ (NS: No significativo)

\begin{tabular}{ccccc}
\hline \multirow{2}{*}{$\begin{array}{c}\text { Nivel de harina de } \\
\text { pulpa de café (\%) }\end{array}$} & \multicolumn{5}{c}{ Evaluación sensorial } \\
\cline { 2 - 5 } & Apariencia & Color & Olor & Sabor \\
\hline 0 & $9.87 \pm 0.38$ & $7.91 \pm 0.39$ & $0.43 \pm 0.17$ & $0.51 \pm 0.12$ \\
5 & $10.16 \pm 0.46$ & $7.63 \pm 0.40$ & $0.31 \pm 0.09$ & $0.46 \pm 0.18$ \\
15 & $10.76 \pm 0.42$ & $9.59 \pm 0.39 *$ & $0.43 \pm 0.16$ & $0.47 \pm 0.16$ \\
25 & $9.29 \pm 0.58$ & $8.95 \pm 0.51$ & $0.50 \pm 0.20$ & $0.68 \pm 0.33$ \\
35 & $9.95 \pm 0.58$ & $8.85 \pm 0.58$ & $0.58 \pm 0.18$ & $0.71 \pm 0.16$ \\
\hline $\begin{array}{c}\text { Nivel de } \\
\text { significancia } \\
\text { Dunnett }\end{array}$ & NS & 0.05 & NS & NS \\
\hline
\end{tabular}

nancia de peso, conversión alimenticia y calidad sensorial de la carne, pero el consumo de alimento se redujo con el incremento de la harina de pulpa de café en la dieta.

- Los mejores rendimientos de carcasa se registraron con dietas de hasta $25 \%$ de inclusión de harina de pulpa de café en el concentrado.

\section{Agradecimientos}

Los autores agradecen al Instituto de Investigación en Ganadería y Biotecnología (IGBI) de la UNTRM por financiar esta investigación. Asimismo, al Mg. Patricio Córdova, al Dr. Wilson M. Castro y al Dr. Raúl Siche por sus aportes y sugerencias en la redacción del presente artículo

\section{Literatura Citada}

1. Apráez JE, Fernández L, Fernández A. 2008. Efecto del empleo de forrajes $\mathrm{y}$ alimentos no convencionales sobre el comportamiento productivo, rendimiento en canal y calidad de la carne de cuyes (Cavia porcellus). Vet Zootecnia 2(2): 29-34.
2. Bautista EO, Pernía J, Barrueta D, Useche M. 2005. Pulpa ecológica de café ensilada en la alimentación de alevines del híbrido cachamay (Colossoma macropomum $x$ Piaractus brachypomus). Rev Científica FCVLUZ 15(1): 33-40.

3. Bazay G, Carcelén F, Ara M, Jiménez R, Gonzáles R, Quevedo W. 2014. Efecto de los manano-oligosacáridos sobre los parámetros productivos de cuyes (Cavia porcellus) durante la fase de engorde. Rev Inv Vet Perú 25: 198204. doi: 10.15381/rivep.v25i2.8491

4. Braham J, Bressani R. 1990. Coffee pulp. Composition, technology and utilization. Ottawa, Canadá: International Development Research Centre. 96 p.

5. Carbajal CS. 2015. Evaluación preliminar de tres alimentos balanceados para cuyes (Cavia porcellus) en acabado en el Valle del Mantaro. Tesis de Ingeniero Zootecnista. Lima, Perú: Univ Nacional Agraria La Molina. $78 \mathrm{p}$

6. Castillo C, Carcelén F, Quevedo W, Ara M. 2012. Efecto de la suplementación con bloques minerales sobre la productividad de cuyes alimentados con forraje. Rev Inv Vet Perú 23: 414-419. doi: 10.15381/rivep.v23i4.946 
7. Char C, Yoplac I, Escalona VH. 2016. Microbiological and functional quality of ready-to-eat arugula as treated by combinations of UV-C and nonconventional modified atmospheres. J Food Process Pres. doi: 10.1111/jfpp. 12978

8. Chauca L. 1997. Producción de cuyes (Cavia porcellus). Estudio FAO producción y sanidad animal 138. Roma, Italia: FAO. 77 p.

9. Clemente E, Arbaiza T, Carcelén F, Lucas O, Bazán V. 2003. Evaluación del valor nutricional de la Puya llatensis en la alimentación del cuy (Cavia porcellus). Rev Inv Vet Perú 14: 1-6. doi: 10.15381/rivep.v14i1.1583

10. [COOPARM] Cooperativa Agraria Rodríguez de Mendoza. 2016. Nuestros productos. [Internet]. Disponible en: http://www.cooparm.org/web/ index3.php

11. Donkoh A, Atuahene CC, Kese AG, Mensah B. 1988. The nutritional value of dried coffee pulp (DCP) in broiler chickens diets. Anim Feed Sci Tech 22: 139-146. doi: 10.1016/0377-8401(88)90081-8

12. Duban J, Gutiérrez N, Oviedo OM. 2012. Uso de subproductos agrícolas en la alimentación de conejos en fases de ceba y reproducción. Biotecnol Sector Agropec Agroind 10(2): 236-242.

13. Ferreira I, Olalquiaga JR, Teixeira JC. 2003. Componentes de carcaça e composição de alguns cortes de cordeiros Texel x Bergamácia, Texel x Santa Inês e Santa Inês puros, terminados em confinamento, com casca de café como parte da dieta. R Bras Zootec 32: 1999-2003. doi: 10.1590/S151635982003000800025

14. Funes CA, Rodríguez J, Arroyo R, Sáenz C, Blanco F. 1986. Evaluación de la pulpa de café deshidratada en po1 los de engorde durante los periodos de iniciación y engorde (0-7 semanas). Uniciencia 3(1): 11-18.

15. Gil W. 2007. Importancia del cuy y su competitividad en el mercado. En: XX Reunión ALPA. Cusco: Asociación Latinoamericana de Producción Animal.
16. [INEI] Instituto Nacional de Estadística e Informática. 2012. IV Censo Nacional Agropecuario (CENAGRO). Lima, Perú. [Internet]. Disponible en: http://censos.inei.gob.pe/Cenagro/ redatam/\#

17. Lozada P, Jiménez R, San Martín F, Huamán A. 2013. Efecto de la inclusión de cebada grano y semilla de girasol en una dieta basada en forraje sobre el momento óptimo de beneficio de cuyes. Rev Inv Vet Perú 24: 25-31. doi: 10.15381/rivep.v24i1.1650

18. Mantilla JA. 2012. Diferenciación reproductiva, productiva y molecular de cuyes nativos de la región Cajamarca. Tesis de Doctor en Ciencias. Cajamarca: Univ Nacional de Cajamarca. 149 p.

19. Mattos J, Chauca L, San Martín F, Carcelén F, Arbaiza T. 2003. Uso del ensilado biológico de pescado en la alimentación de cuyes mejorados. Rev Inv Vet Perú 14: 89-96. doi: 10.15381/ rivep.v14i2.1612

20. Meza GA, Cabrera RP, Morán JJ, Meza FF, Cabrera CA, Meza CJ, Meza JS, et al. 2014. Mejora de engorde de cuyes (Cavia porcellus L) a base de gramíneas y forrajeras arbustivas tropicales en la zona de Quevedo, Ecuador. Idesia 32: 75-80. doi: 10.4067/ S0718-34292014000300010

21. [MINAGRI] Ministerio de Agricultura y Riego. 2003. Situación de las actividades de crianza y producción-Cuyes. Lima, Perú. [Internet]. Disponible en: http://www.minagri.gob.pe/portal/objetivos/40-sector-agrario/situacion-de-lasactividades-de-crianza-y-producci/300cuyes? start $=1$

22. Montes IT. 2012. Guía técnica: Asistencia técnica dirigida a la crianza tecnificada de cuyes. Cajamarca, Perú. [Internet]. Disponible en: http:// www.agrobanco.com.pe/data/uploads/ ctecnica/015-a-crianza-tecnificada.pdf

23. Morales A, Carcelén F, Ara M, Arbaiza T, Chauca L. 2011. Evaluación de dos niveles de energía en el comportamiento productivo de cuyes (Cavia 
porcellus) de la raza Perú. Rev Inv Vet Perú 22: 177-182. doi: 10.15381/ rivep.v22i3.254

24. Moreau Y, Arredondo J, Perraud I, Roussos S. 2003. Utilización dietética de la proteína y de la energía de la pulpa de café fresca y ensilada por las tilapias del Nilo (Oreochromis niloticus). Braz Arch Biol Technol 46(2): 35-347.

25. Noriega A, Silva R, García M. 2008. Utilización de la pulpa de café en la alimentación animal.Zootec Trop 26:411-419.

26. Noriega A, Silva R, García M. 2009. Composición química de la pulpa de café a diferentes tiempos de ensilaje para su uso potencial en la alimentación animal. Zootec Trop 27: 135-141.

27. Quintana E, Jiménez, R, Carcelén F, San Martín F, Ara M. 2013. Efecto de dietas de alfalfa verde, harina de cebada y bloque mineral sobre la eficiencia productiva de cuyes. Rev Inv Vet Perú 24: 425-432. doi: 10.15381/rivep.v24i4. 2744

28. Ramirez JR. 1988. Phenolic compounds in coffee pulp: quantitative determination by HPLC. J Sci Food Agric 43: 135-144. doi: 10.1002/jsfa. 274043-0204
29. Ramos L, Chamorro E, Benavides JP. 2013. Evaluación de harina de nabo (Brassica campestris) en alimentación de cuyes (Cavia porcellus). Invest Pec 2(2): 33-40.

30. Rodríguez J, Aguirre D, Benavides $O$. 1994. Efecto de cuatro niveles de pulpa de café en alimentación de gallinas ponedoras (iniciación-desarrollo). Uniciencia 11: 23-35.

31. Sánchez A, Sánchez S, Godoy S, Díaz $R$, Vega N. 2009. Gramíneas tropicales en el engorde de cuyes mejorados sexados (Cavia porcellus linnaeus) en la zona de La Maná. Cienc Tecnol 2: 25-28.

32. Sánchez A, Zambrano D, Torres E, Meza G 2012. Forrajeras tropicales y banano maduro (Musa paradisiaca) en el engorde de cuyes (Cavia porcellus L) en el Cantón Quevedo. Actas Iberoam Conserv Anim 2: 287-290.

33. Vivas JA. 2013. Especies alternativas: manual de crianza de cobayos (Cavia porcellus). Nicaragua: Universidad Nacional Agraria. 47 p. [Internet]. Disponible en: http://repositorio.una.edu.ni/ 2472/1/RENL01V856.pdf 